\title{
Phase 1 study of efatutazone, a novel oral peroxisome proliferator-activated receptor gamma agonist, in combination with FOLFIRI as second-line therapy in patients with metastatic colorectal cancer
}

\author{
Yoshito Komatsu • Takayuki Yoshino • Kentaro Yamazaki • \\ Satoshi Yuki • Nozomu Machida • Takahide Sasaki • \\ Ichinosuke Hyodo • Yutaka Yachi • Hiroshi Onuma • Atsushi Ohtsu
}

Received: 24 October 2013 / Accepted: 1 December 2013 /Published online: 15 December 2013

(C) The Author(s) 2013. This article is published with open access at Springerlink.com

Summary Background Efatutazone, a novel oral highlyselective peroxisome proliferator-activated receptor gamma (PPAR $\gamma)$ agonist, has demonstrated some inhibitory effects on disease stabilization in patients with metastatic colorectal cancer (mCRC) enrolled in previous phase I studies. Here, we evaluate the safety and pharmacokinetics of efatutazone combined with

A summary of this study has been partly presented at the 37th European Society of Medical Oncology Congress 2012.

Clinical Trial Registration ID: JapicCTI-101230

Y. Komatsu $(\varangle) \cdot$ T. Sasaki

Hokkaido University Hospital Cancer Center, Kita 14 Nishi 5,

Kita-ku, Sapporo, Hokkaido 060-8648, Japan

e-mail: ykomatsu@ac.cyberhome.ne.jp

T. Yoshino $\cdot$ T. Sasaki

Department of Gastroenterology and Gastrointestinal Oncology,

National Cancer Center Hospital East, Kashiwa, Japan

K. Yamazaki $\cdot$ N. Machida

Division of Gastrointestinal Oncology, Shizuoka Cancer Center,

Shizuoka, Japan

S. Yuki

Department of Gastroenterology and Hepatology, Hokkaido

University Hospital, Sapporo, Japan

I. Hyodo

Division of Gastroenterology, Graduate School of Comprehensive Human Sciences, University of Tsukuba, Ibaraki, Japan

Y. Yachi $\cdot$ H. Onuma

Clinical Developmental Department II, Daiichi Sankyo Co., Ltd.,

Tokyo, Japan

A. Ohtsu

Exploratory Oncology Research \& Clinical Trial Center, National

Cancer Center Hospital East, Kashiwa, Japan
FOLFIRI (5-fluorouracil, levo-leucovorin, and irinotecan) as second-line chemotherapy in Japanese patients with mCRC. Methods Dose-limiting toxicities (DLTs) were evaluated at 2 efatutazone dose levels of 0.25 and $0.50 \mathrm{mg}$ (the recommended dose $[R D]$ of efatutazone monotherapy) twice daily in combination with FOLFIRI in a 3-9 patient cohort. Furthermore, tolerability at the RD level was assessed in additional patients, up to 12 in total. Blood samples for pharmacokinetics and biomarkers and tumor samples for archival tissues were collected from all patients. Results Fifteen patients $(0.25 \mathrm{mg}, 3 ; 0.5 \mathrm{mg}$, 12) were enrolled. No DLTs were observed. Most patients experienced weight increase $(100 \%)$ and edema $(80.0 \%)$, which were manageable with diuretics. Common grade 3/4 toxicities were neutropenia (93.3\%), leukopenia ( $46.7 \%$ ), and anemia (33.3\%). Stable disease was observed in 8 of the 14 patients evaluable for tumor response. The plasma adiponectin levels increased over time and increased dose. No clear relationship was detected between treatment efficacies and plasma levels of adiponectin as well as the expression levels of PPAR $\gamma$ and the retinoid $\mathrm{X}$ receptor in tumor tissues. Conclusions Efatutazone combined with FOLFIRI demonstrates an acceptable safety profile and evidence of disease stabilization in Japanese patients with mCRC. The RD for efatutazone monotherapy can be used in combination with FOLFIRI.

Keywords Efatutazone $\cdot$ Peroxisome proliferator-activated receptor gamma $\cdot$ FOLFIRI $\cdot$ Colorectal cancer

\section{Introduction}

Peroxisome proliferator-activated receptor gamma $(\operatorname{PPAR} \gamma)$ is a member of the nuclear hormone receptor 
superfamily. It is activated through its ligands and is involved in the regulation of inflammation, cell cycle progression, cell proliferation, apoptosis, carcinogenesis, and angiogenesis [1-4]. Preclinical studies demonstrated synergistic or additive effects of PPAR $\gamma$ ligands with chemotherapeutic agents on cancer cell apoptosis and growth inhibition, and suggested their potential clinical use in cancer therapy [4-7].

Efatutazone, a novel oral highly-selective thiazolidinedione PPAR $\gamma$ agonist, shows greater potency than second-generation thiazolidinediones such as pioglitazone [8]. In preclinical tumor models, proliferation of human anaplastic thyroid carcinoma and pancreatic tumor cell lines were inhibited in vitro, and human colorectal cancer (CRC) and anaplastic thyroid carcinoma cell xenografts were inhibited in nude rodents $[9,10]$. PPAR $\gamma$ activity-related adiponectin is also considered a useful biomarker of carcinogenesis and progression of colorectal adenoma [11].

A US Phase 1 study of efatutazone monotherapy in patients with advanced solid malignancies demonstrated acceptable safety and evidence of antitumor activity [12]. A similar study in Japanese patients with metastatic solid tumors confirmed the results (presented in a poster session at the $36^{\text {th }}$ Congress of the European Society for Medical Oncology, 2011) that efatutazone is potentially effective against CRC, by achieving sustained disease stabilization.

In the clinical setting, FOLFOX (5-fluorouracil, levoleucovorin, and oxaliplatin), CapeOX (capecitabine and oxaliplatin) and FOLFIRI (5-fluorouracil, levo-leucovorin, and irinotecan) regimens are global standard therapies for CRC, demonstrating efficacy and tolerability, and are often chosen as first- and second-line therapies, respectively [13, 14]. The combination of PPAR $\gamma$ ligands with existing chemotherapy is reported to be beneficial for cancer prevention and therapy [4-7], and a novel combination with greater clinical efficacy against advanced refractory tumors including metastatic CRC (mCRC) is needed.

This Phase 1 study was designed to evaluate the safety profile and pharmacokinetics of efatutazone in combination with FOLFIRI as second-line therapy in Japanese patients with mCRC. Secondary objectives included assessment of the preliminary antitumor efficacy of efatutazone, assessment of potential biomarkers of efatutazone including plasma adiponectin, and to determine the recommended dose (RD) of efatutazone in combination with FOLFIRI.

\section{Materials and methods}

\section{Study design}

This Phase 1, open-label, dose-escalation study was conducted at 3 medical centers in Japan between September
2010 and June 2012 (JapicCTI-101230; Clinical Trials Information/JapicCTI, http://www.clinicaltrials.jp/user/ cteSearch_e.jsp). It was performed in accordance with the International Conference on Harmonisation Good Clinical Practice Guidelines, the principle of the Declaration of Helsinki and all applicable laws and regulations in Japan. The protocol was reviewed and approved by the Institutional Review Boards of all participating study sites. All patients provided written informed consent before enrollment.

The dose-escalation study had a $3+3$ design and 2 steps. Since previous Phase 1 studies had indicated an RD of $0.50 \mathrm{mg}$ twice daily (BID) for 4 weeks, we assessed 2 doses of efatutazone, namely, 0.25 and $0.50 \mathrm{mg}$ BID for 4 weeks (1 cycle) in combination with FOLFIRI (irinotecan $180 \mathrm{mg}$ / $\mathrm{m}^{2}$ intravenous [IV] infusion over 90 min or longer, levoleucovorin $200 \mathrm{mg} / \mathrm{m}^{2} \mathrm{IV}$ infusion over $120 \mathrm{~min}$, and 5fluorouracil IV continuous infusion $2400 \mathrm{mg} / \mathrm{m}^{2}$ over $46 \mathrm{~h}$ ) once every 2 weeks, in 3 to 9 patients each in Step 1 (doseescalation phase for evaluation of dose-limiting toxicity [DLT]).

DLTs were defined as follows: (a) grade 3 or higher neutropenia complicated by fever $\geq 38.5^{\circ} \mathrm{C}$ or infection, or grade 4 neutropenia with a duration of 7 days or longer; (b) grade 4 thrombocytopenia, or grade 3 thrombocytopenia requiring transfusion; (c) grade 4 anemia; (d) grade 3 or higher pleural or pericardial effusion, peripheral edema or ascites unresponsive to treatment; (e) uncontrollable grade 3 or higher severe fatigue, anorexia, nausea, vomiting or diarrhea, despite maximal supportive therapy; (f) any other grade 3 or higher toxicities than those described in definitions (d) and (e), except for fever without neutropenia and transient electrolyte abnormality.

According to the protocol, if 1 of the first 3 patients at a dose level would experience a DLT, 3 more patients would be enrolled. In case of no DLTs in 3 patients or a maximum of 1 DLT in 6 patients, the study would proceed to the next dose level. In case of 2 DLTs in 6 patients, another 3 patients would be enrolled. RD was based on the Step 1 safety data, and would be administered in combination with FOLFIRI to up to 9 additional patients in Step 2. Administration of efatutazone and FOLFIRI would continue until disease progression or unacceptable toxicity was observed, and then efatutazone and FOLFIRI doses would be reduced based on set guidelines.

\section{Study population}

Inclusion criteria were: age $\geq 20$ years; histologically/ cytologically confirmed mCRC after failure of first-line chemotherapy; an Eastern Cooperative Oncology Group (ECOG) performance status score of 0 or 1 ; a 
Table 1 Baseline characteristics (Safety analysis set)

\begin{tabular}{|c|c|c|c|}
\hline \multirow[t]{2}{*}{ Characteristic } & \multicolumn{3}{|c|}{ Treatment cohort } \\
\hline & $\begin{array}{l}0.25 \mathrm{mg} \text { BID } \\
(n=3)\end{array}$ & $\begin{array}{l}0.50 \mathrm{mg} \text { BID } \\
(n=12)\end{array}$ & $\begin{array}{l}\text { Overall } \\
(n=15)\end{array}$ \\
\hline Median age in years & 63 & 64 & 63 \\
\hline (range) & $(56-64)$ & $(41-73)$ & $(41-73)$ \\
\hline \multicolumn{4}{|l|}{ Gender } \\
\hline Male & $1(33.3)$ & $6(50.0)$ & $7(46.7)$ \\
\hline Female & $2(66.7)$ & $6(50.0)$ & $8(53.3)$ \\
\hline \multicolumn{4}{|l|}{ ECOG performance status } \\
\hline 0 & $2(66.7)$ & $8(66.7)$ & $10(66.7)$ \\
\hline 1 & $1(33.3)$ & $4(33.3)$ & $5(33.3)$ \\
\hline \multicolumn{4}{|l|}{ Primary site } \\
\hline Rectum & $3(100.0)$ & $7(58.3)$ & $10(66.7)$ \\
\hline Colon & 0 & $4(33.3)$ & $4(26.7)$ \\
\hline Colon and rectum & 0 & $1(8.3)$ & $1(6.7)$ \\
\hline \multicolumn{4}{|l|}{ Histological type } \\
\hline Well differentiated & $2(66.7)$ & $4(33.3)$ & $6(40.0)$ \\
\hline Moderately differentiated & $1(33.3)$ & $6(50.0)$ & $7(46.7)$ \\
\hline Poorly differentiated & 0 & $1(8.3)$ & $1(6.7)$ \\
\hline Others & 0 & $1(8.3)$ & $1(6.7)$ \\
\hline \multicolumn{4}{|l|}{ Previous chemotherapy } \\
\hline Oxaliplatin-based regimen & $2(66.7)$ & $1(8.3)$ & $3(20.0)$ \\
\hline Oxaliplatin-based regimen + bevacizumab & 0 & $11(91.7)$ & $11(73.3)$ \\
\hline Capecitabine monotherapy & $1(33.3)$ & 0 & $1(6.7)$ \\
\hline \multicolumn{4}{|l|}{ UGT1A1 genotype } \\
\hline Wild $(* 1 / * 1)$ & $1(33.3)$ & $7(58.3)$ & $8(66.7)$ \\
\hline Heterozygous $(* 1 / * 28, * 1 / * 6)$ & $1(33.3)$ & $4(33.3)$ & $5(33.3)$ \\
\hline Homozygous $(* 28 / * 28, * 6 / * 6, * 28 / * 6)$ & $1(33.3)^{\mathrm{a}}$ & $1(8.3)^{\mathrm{b}}$ & $2(13.3)$ \\
\hline
\end{tabular}

Values represent the number $(\%)$ of subjects

BID twice daily, ECOG Eastern Cooperative Oncology Group, $n$ number of subjects

${ }^{\text {a }}$ One subject had UGT1A1*6/*6 genotype

${ }^{\mathrm{b}}$ One subject had UGT1A1 *28/ *28 genotype
Efficacy assessment

Computed tomography or magnetic resonance imaging was performed every 2 cycles for efficacy assessment.

Tumor response was evaluated according to the Response Evaluation Criteria in Solid Tumors, Version 1.1. Overall response rate (ORR) and disease control rate (DCR) were calculated. Progression-free survival (PFS) was defined as time from enrollment to the detection of progressive disease (PD) or death (or the date of last tumor evaluation in stable patients).

\section{Pharmacokinetics}

Blood samples were collected on Day 1 (Cycles 1 and 2) at the following time points: predose (immediately before the morning dose of efatutazone) and postdose $(0.5,1,2,3,4,6$ and 8 to $10 \mathrm{~h})$. Additional samples were collected predose on Days 8 and 15 (Cycle 1). Validated liquid chromatography-tandem mass spectrometry was used to measure the plasma concentration of the free form of efatutazone. The plasma concentration 
Table 2 Summary of treatmentemergent adverse events that occurred in 3 or more patients throughout the study (Safety analysis set)
Values represent the number $(\%)$ of subjects

MedDRA Medical Dictionary for Regulatory Activities

\begin{tabular}{|c|c|c|c|c|}
\hline \multirow[t]{2}{*}{ MedDRA Preferred term } & \multicolumn{2}{|c|}{ Treatment-emergent } & \multicolumn{2}{|c|}{ Efatutazone-related } \\
\hline & Overall & Grade 3 or higher & Overall & Grade 3 or higher \\
\hline \multicolumn{5}{|l|}{ Hematotoxicity } \\
\hline Neutropenia & $15(100.0)$ & $14(93.3)$ & $7(46.7)$ & $6(40.0)$ \\
\hline Leukopenia & $14(93.3)$ & $7(46.7)$ & $6(40.0)$ & $2(13.3)$ \\
\hline Anemia & $13(86.7)$ & $5(33.3)$ & $11(73.3)$ & $4(26.7)$ \\
\hline Thrombocytopenia & $10(66.7)$ & $1(6.7)$ & $3(20.0)$ & $1(6.7)$ \\
\hline \multicolumn{5}{|l|}{ Non-hematotoxicity } \\
\hline Weight increase & $15(100.0)$ & 0 & $15(100.0)$ & 0 \\
\hline Edema & $12(80.0)$ & $1(6.7)$ & $10(66.7)$ & $1(6.7)$ \\
\hline Nausea & $9(60.0)$ & 0 & 0 & 0 \\
\hline Vomiting & $8(53.3)$ & 0 & 0 & 0 \\
\hline Alopecia & $8(53.3)$ & 0 & 0 & 0 \\
\hline Fatigue & $8(53.3)$ & $1(6.7)$ & $3(20.0)$ & $1(6.7)$ \\
\hline Decreased appetite & $7(46.7)$ & $1(6.7)$ & $2(13.3)$ & 0 \\
\hline Diarrhea & $7(46.7)$ & 0 & $1(6.7)$ & 0 \\
\hline Hypoalbuminemia & $6(40.0)$ & $2(13.3)$ & $2(13.3)$ & 0 \\
\hline Constipation & $5(33.3)$ & 0 & $3(20.0)$ & 0 \\
\hline Blood alkaline phosphatase increased & $5(33.3)$ & $1(6.7)$ & 0 & 0 \\
\hline Blood creatinine increased & $5(33.3)$ & 0 & $1(6.7)$ & 0 \\
\hline Hypercholesterolemia & $4(26.7)$ & $1(6.7)$ & $3(20.0)$ & 0 \\
\hline Hyponatremia & $4(26.7)$ & $1(6.7)$ & $4(26.7)$ & $1(6.7)$ \\
\hline Abdominal pain & $4(26.7)$ & $1(6.7)$ & $2(13.3)$ & 0 \\
\hline Pyrexia & $4(26.7)$ & 0 & $1(6.7)$ & 0 \\
\hline $\begin{array}{l}\text { Gamma-glutamyltransferase } \\
\text { increased }\end{array}$ & $4(26.7)$ & $2(13.3)$ & 0 & 0 \\
\hline Nasopharyngitis & $3(20.0)$ & 0 & $1(6.7)$ & 0 \\
\hline Stomatitis & $3(20.0)$ & 0 & $1(6.7)$ & 0 \\
\hline Malaise & $3(20.0)$ & $1(6.7)$ & $2(13.3)$ & $1(6.7)$ \\
\hline Aspartate aminotransferase increased & $3(20.0)$ & 0 & 0 & 0 \\
\hline
\end{tabular}

of an active metabolite of irinotecan ( $\mathrm{SN}-38)$ was also measured immediately after irinotecan hydrochloride (CPT-11) administration in the morning on Day 1 (Cycles 1 and 2).

\section{Biomarkers}

Blood samples for the measurement of plasma adiponectin were collected predose on Days 1, 8 and 15 in Cycle 1, and Day 1 in Cycles 2, 3 and 4. Plasma adiponectin concentrations were determined by quantitative sandwich enzyme immunoassay kit (Quantikine ${ }^{\circledR}, \mathrm{R} \& D$ systems, Inc., Minneapolis, $\mathrm{MN}$, the USA).

Expression levels of PPAR $\gamma$ and retinoid $\mathrm{X}$ receptor (RXR) in archived tumor specimens were studied by immunohistochemistry using PPAR $\gamma$ (C26H12) Rabbit mAb (Cell Signaling Technology, Inc., Danvers, MA, the USA) and Rabbit Anti-Human Retinoic X Receptor Gamma Polyclonal Antibody (Spring Bioscience, Inc., Pleasanton, CA, the USA), respectively.

\section{Results}

Patient demographics

A total of 15 patients were enrolled and received efatutazone treatment in combination with FOLFIRI. In Step 1, 2 cohorts with 3 patients each received efatutazone at 0.25 and $0.50 \mathrm{mg}$ BID, respectively. In Step 2, an additional 9 patients received efatutazone $0.50 \mathrm{mg}$ BID.

Enrollment started in September 2010, and 15 patients were enrolled by March 2012. Baseline patient characteristics are summarized in Table 1. UGT1A1 genotypes, included 1 homozygous *6/*6 genotype, and 1 homozygous $* 28 / * 28$ genotype.

Safety

Overall, efatutazone showed acceptable safety at both 0.25 and $0.50 \mathrm{mg}$ BID. No DLTs were observed during the 
Fig. 1 Waterfall plot of the best percent changes from baseline in the target lesion. Best percent change from baseline (\%) in the target lesion $=([$ the minimum sum of the longest diameters at all measurement time points - the sum of the longest diameters at baseline] / [the sum of the longest diameters at baseline] $) \times 100$. BID twice daily, $P D$ progressive disease, $S D$ stable disease

Fig. 2 Concentration-time curve of the free form of efatutazone following oral dosing on Day 1 in Cycle 1 (single dose [a]) and Day 1 in Cycle 2 (repeated dose [b]). $B I D$ twice daily

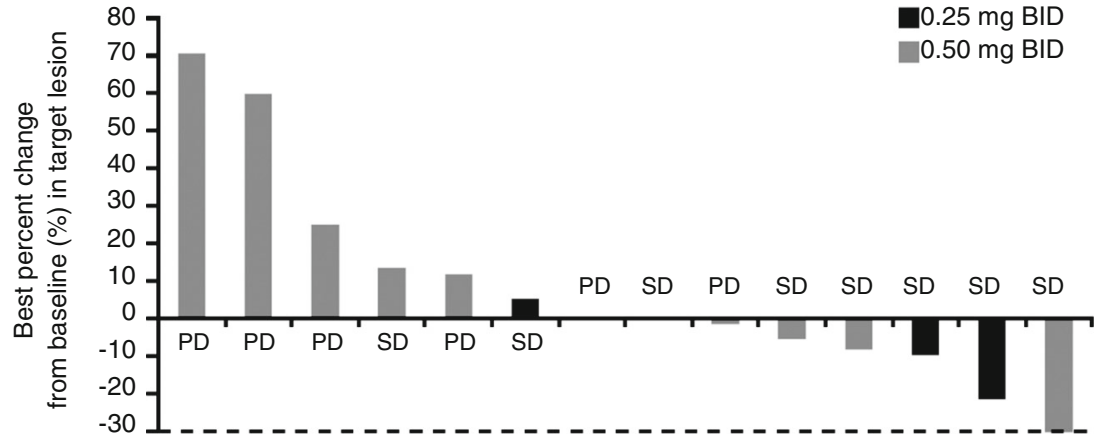

$\mathrm{SD}, \mathrm{PD}$ : Best overall response
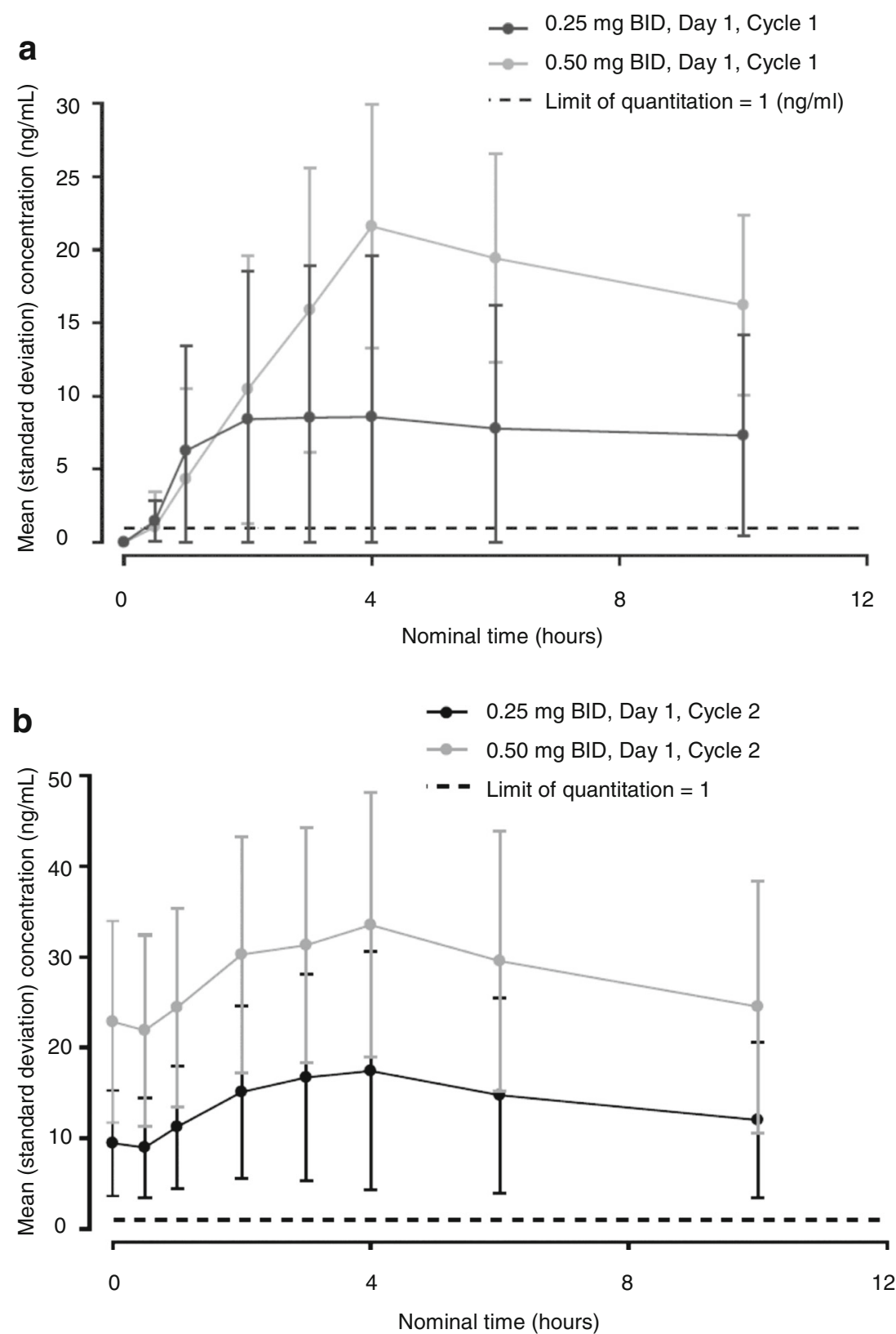
Table 3 Summary of pharmacokinetic parameters (Pharmacokinetic analysis set)

\begin{tabular}{|c|c|c|c|c|}
\hline \multirow[t]{2}{*}{ Parameter } & \multicolumn{2}{|l|}{$0.25 \mathrm{mg}$ BID } & \multicolumn{2}{|l|}{$0.50 \mathrm{mg}$ BID } \\
\hline & Day 1 in Cycle $1^{\mathrm{a}}(n=3)$ & Day 1 in Cycle $2^{\mathrm{b}}(n=3)$ & Day 1 in Cycle $1^{\mathrm{a}}(n=12)$ & Day 1 in Cycle $2^{\mathrm{b}}(n=6)$ \\
\hline $\mathrm{C}_{\max }(\mathrm{ng} / \mathrm{ml})$ & $10.1(9.5)$ & $18.3(13.2)$ & $22.2(7.97)$ & $35.5(14.7)$ \\
\hline $\mathrm{T}_{\max }$ (hours) & $4.03(2.00-8.03)$ & $3.25(1.98-3.95)$ & $4.08(1.97-6.00)$ & $2.99(1.97-4.03)$ \\
\hline $\mathrm{AUC}_{\text {last }}(\mathrm{ng} \mathrm{h} / \mathrm{ml})$ & $60.9(66.9)$ & $125(85.0)$ & $127(52.9)$ & $255(105)$ \\
\hline $\mathrm{AUC}_{\text {tau }}(\mathrm{ng} \mathrm{h} / \mathrm{ml})$ & $116(99.3)$ & $159(110)$ & $193(66.5)$ & $325(154)$ \\
\hline $\mathrm{C}_{\text {trough }}(\mathrm{ng} / \mathrm{ml})$ & - & $8.68(5.91)$ & - & $21.1(10.8)$ \\
\hline
\end{tabular}

For $\mathrm{C}_{\max }, \mathrm{AUC}_{\text {last, }} \mathrm{AUC}_{\text {tau, }}$ and $\mathrm{C}_{\text {trough }}$, values represent the means (standard deviation)

For $\mathrm{T}_{\text {max }}$, values represent the median (range)

$A U C_{\text {last }}$ area under the concentration-time curve from zero to the last quantifiable concentration, $A U C_{\text {tau }}$ area under the concentration-time curve during the dosing interval, $B I D$ twice daily, $C_{\max }$ maximum plasma concentration, $C_{\text {trough }}$ trough plasma concentration, $n$ number of subjects, $T_{\text {max }}$ time to reach the maximum plasma concentration

${ }^{a}$ Following single-dose administration

${ }^{\mathrm{b}}$ Following repeated-dose administration

evaluation period (Cycle 1, Step 1). The median duration (range) of efatutazone treatment was 152.0 (71-157) days in the $0.25 \mathrm{mg}$ BID group, 62.5 (21-241) days in the $0.50 \mathrm{mg}$ BID group, and 67.0 (21-241) days in the overall study population.

Treatment-emergent AEs (TEAEs) that occurred in 3 or more patients throughout the study period are summarized in Table 2. Most patients experienced weight increase (100\%) and edema $(80.0 \%)$, usually at a severity of $\leq$ grade 2 . These were managed with diuretics. Common grade $3 / 4$ toxicities were neutropenia (93.3\%), leukopenia (46.7 \%) and anemia $(33.3 \%)$, and were managed with supportive therapy and/or FOLFIRI modification.

A total of $14(93.3 \%)$ patients experienced at least 1 grade 3 or more severe TEAE: $3(100 \%)$ in the 0.25 BID group, and $11(91.7 \%)$ in the $0.50 \mathrm{mg}$ BID groups. In the $0.25 \mathrm{mg}$ BID group, 1 patient experienced grade 3 edema (44 days after the first administration of efatutazone), which was related to efatutazone and recovered with diuretics and temporary discontinuation of efatutazone. No patients experienced grade 3 or more severe weight increase. Five patients (including 2 patients with an UGT1A1 homozygous genotype) experienced grade 4 neutropenia $(<7$ days duration), and 1 patient experienced grade 4 thrombocytopenia and grade 4 anemia.

No deaths were reported throughout the study period. Five patients $(33.3 \%)$ discontinued the study due to efatutazonerelated TEAEs (fatigue, bronchitis, edema, anemia, and interstitial pneumonia). Three patients $(20.0 \%)$ experienced serious TEAEs related to both efatutazone and FOLFIRI, but no serious TEAEs during the DLT evaluation period. One patient on $0.25 \mathrm{mg}$ BID had grade 3 fatigue and grade 2 bronchitis on Day 71 . The study drugs were discontinued, and the patient received oxygen inhalation and antimicrobials, recovered of the fatigue and had relief of the bronchitis by Day 79. One patient on $0.50 \mathrm{mg}$ BID had grade 3 interstitial pneumonia on Day 35. The study drugs were discontinued, steroid pulse therapy and antimicrobials were administered, and the patient experienced relief of the interstitial pneumonia by Day 55 . One patient on $0.50 \mathrm{mg}$ BID had grade 3 febrile neutropenia on Day 36, and grade 4 anemia and grade 4 neutropenia on Day 37. The patient received platelet transfusion, antimicrobials and granulocyte colony-stimulating factor for the anemia. Fever improved by Day 40 and anemia and neutropenia by Day 43 .

\section{Efficacy}

A total of 14 patients were evaluable for efficacy analysis, with ORR of $0 \%$, and DCR of $57.1 \%$ (95\% confidence interval [CI]: $28.9,82.3)$. Five (45.5\%) of 11 patients on efatutazone $0.50 \mathrm{mg}$ BID had stable disease (SD). A waterfall

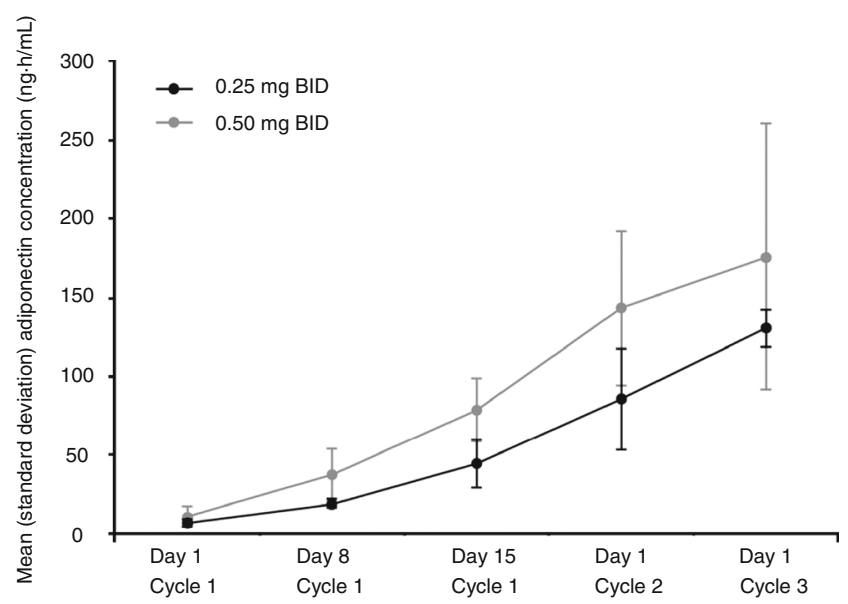

Fig. 3 Time course of plasma adiponectin levels. BID twice daily 
plot of the best percentage changes from baseline in the target lesion is shown in Fig. 1.

Median (95\% CI) PFS was 158.5 (158-159) days in the $0.25 \mathrm{mg}$ BID group, 73.0 (65-235) days in the $0.50 \mathrm{mg}$ BID group, and $85.0(70-159)$ days in the overall study population.

\section{Pharmacokinetics}

Plasma concentration-time curves following administration on Day 1 (Cycles 1 and 2) are shown in Fig. 2. Although an absorption lag usually followed single-dose administration in the $0.50 \mathrm{mg}$ cohort, the mean plasma concentration of efatutazone generally increased with repeated doses.

Pharmacokinetic values of the free form of efatutazone following oral administration on Day 1 (Cycles 1 and 2) are shown in Table 3. The $\mathrm{C}_{\max }$ and $\mathrm{AUC}_{\text {tau }}$ in patients treated with efatutazone $0.50 \mathrm{mg}$ were approximately 2 times those in patients treated with efatutazone $0.25 \mathrm{mg}$.

The trough plasma concentration did not increase with repeated doses.

\section{Biomarkers}

The plasma adiponectin levels increased over time and increased dose as shown in Fig. 3.

There were no apparent differences in the PPAR $\gamma$ expression levels in archived tumor specimens between patients with $\mathrm{SD}$ and those with PD (Mean [range] $\mathrm{H}$ scores in nucleus, 106.4 [5-180] versus 155.8 [5-240]; H score $=\sum$ [the staining intensity $\times$ the occupied percentage of positive cells]). RXR expression levels were not significantly different in the nucleus $\mathrm{H}$ scores between patients with SD and those with PD (20.0 [0-70] versus $25.0[0-75])$.

\section{Discussion}

This is the first study of efatutazone combined with FOLFIRI evaluating the safety profile and pharmacokinetics of efatutazone as second-line therapy in Japanese patients with mCRC. The safety profile in this study is consistent with that in previous Phase 1 studies (Ref. [12] and the poster presentation mentioned in the "Introduction"). Compared with the toxicity data of FOLFIRI as second-line chemotherapy for mCRC reported in a FIRIS (IRIS [irinotecan plus S-1] versus FOLFIRI) study [14], the toxicity profile of efatutazone plus FOLFIRI was almost identical to that of FOLFIRI alone, adverse effects including hematotoxicity were more frequent with efatutazone combined with FOLFIRI than with FOLFIRI alone. This higher incidences of adverse effects may partly be attributable to the initial irinotecan IV infusion dose $\left(180 \mathrm{mg} / \mathrm{m}^{2}\right)$ administered in this study, which is rather high for ethnic Japanese patients. In the FIRIS study, irinotecan $150 \mathrm{mg} / \mathrm{m}^{2}$ IV infusion was used [14]. Most adverse effects, including hematotoxicity, were manageable with supportive therapy and discontinuation/modification of efatutazone and/or FOLFIRI.

Preliminary efficacy results appeared to be comparable with those of second-line FOLFIRI therapy for advanced CRC (4 \% PR, $30 \% \mathrm{SD})$ reported in a randomized GERCOR study (randomized control study with FOLFIRI and FOLFOX) [13]. In a previous US Phase 1 study in 27 patients with advanced solid malignancies, 1 (3.7 \%) patient with myxoid liposarcoma achieved sustained PR (690 days on treatment), and 10 (37.0\%) patients had SD for $\geq 60$ days [12]. In a Japanese Phase 1 study in 13 patients with metastatic solid tumors, $1(7.7 \%)$ patient with thymic cancer achieved unconfirmed PR ( $>210$ days on treatment), and 3 (23.1\%) patients had SD (75-170 days on treatment) (the poster presentation mentioned in the "Introduction"). Based on the efficacy results of the present and previous Phase 1 studies, efatutazone is a potential clinically useful anticancer drug.

Efatutazone did not affect plasma concentrations of SN38 (data not shown). Adiponectin is secreted by adipocytes in response to PPAR $\gamma$ agonist-induced gene expression in humans and rodents [15]. Since the plasma adiponectin level in colorectal adenoma patients is significantly lower than age-, sex-, and body mass index-matched non-cancer controls, it is considered a good biomarker for carcinogenesis and progression of colorectal adenoma [11]. Patients whose plasma adiponectin concentration reached $\geq 100 \mu \mathrm{g} /$ $\mathrm{mL}$ tended to have longer PFS than patients with adiponectin $<100 \mu \mathrm{g} / \mathrm{mL}$ (mean [range] PFS, 106.9 [26235] versus 60.6 [36-76] days). The clinical relevance of increased adiponectin levels and efatutazone treatment has to be studied further.

In a US Phase 1 study, archived tumor specimens of patients with SD or PR showed significantly higher PPAR $\gamma$ and RXR expression than those with PD [12]. In the present study, such differences could not be confirmed.

In conclusion, efatutazone at doses of 0.25 and $0.50 \mathrm{mg}$ BID in combination with FOLFIRI demonstrates an acceptable safety profile and evidence of disease control in Japanese patients with $\mathrm{mCRC}$. The RD in combination with FOLFIRI is $0.50 \mathrm{mg}$ BID. The results of a randomized Phase 2 study of efatutazone in combination with FOLFIRI compared with FOLFIRI alone will also be reported soon.

Acknowledgments We are grateful to all the patients who participated in this study. We also thank the members of the Efficacy Safety Evaluation Committee: Hideo Baba, Wataru Ichikawa, and Hiroya Takiuchi.

Funding Daiichi Sankyo Co., Ltd., Japan provided the grant and drugs for this study. 
Ethical standards The clinical research described in this manuscript complies with all applicable international laws and national laws of Japan, where this research was conducted.

Conflicts of interest statement Yoshito Komatsu has received research funding and honoraria from Daiichi Sankyo Co., Ltd. Takayuki Yoshino, KentaroYamazaki, and Nozomu Machida have received research funding from Daiichi Sankyo Co., Ltd. Ichinosuke Hyodo and Atsushi Ohtsu have received consulting fees and honoraria from Daiichi Sankyo Co., Ltd. Yutaka Yachi and Hiroshi Onuma are employees of Daiichi Sankyo Co., Ltd. Satoshi Yuki and Takahide Sasaki have declared no conflicts of interest.

Open Access This article is distributed under the terms of the Creative Commons Attribution License which permits any use, distribution, and reproduction in any medium, provided the original author(s) and the source are credited.

\section{References}

1. Nemenoff RA, Weiser-Evans M, Winn RA (2008) Activation and molecular targets of peroxisome proliferator-activated receptorgamma ligands in lung cancer. PPAR Res 2008:156875

2. Ondrey F (2009) Peroxisome proliferator-activated receptor gamma pathway targeting in carcinogenesis: implications for chemoprevention. Clin Cancer Res 15:2-8

3. Burgermeister E, Seger R (2008) PPARgamma and MEK interactions in cancer. PPAR Res 2008:309469

4. Zou B, Qiao L, Wong BC (2009) Current understanding of the role of PPAR $\gamma$ in gastrointestinal cancers. PPAR Res 2009:816957

5. Skelhorne-Gross G, Nicol CJ (2012) The key to unlocking the chemotherapeutic potential of PPARgamma ligands: having the right combination. PPAR Res 2012:946943

6. Girnun GD, Naseri E, Vafai SB, Qu L, Szwaya JD, Bronson R, Alberta JA, Spiegelman BM (2007) Synergy between PPARgamma ligands and platinum-based drugs in cancer. Cancer Cell 11:395-406

7. Tikoo K, Kumar P, Gupta J (2009) Rosiglitazone synergizes anticancer activity of cisplatin and reduces its nephrotoxicity in 7, 12dimethyl benz $\{\mathrm{a}\}$ anthracene (DMBA) induced breast cancer rats. BMC Cancer 9:107

8. Chen L, Bush CR, Necela BM, Su W, Yanagisawa M, Anastasiadis PZ, Fields AP, Thompson EA (2006) RS5444, a novel PPARgamma agonist, regulates aspects of the differentiated phenotype in nontransformed intestinal epithelial cells. Mol Cell Endocrinol 251: $17-32$

9. Copland JA, Marlow LA, Kurakata S, Fujiwara K, Wong AK, Kreinest PA, Williams SF, Haugen BR, Klopper JP, Smallridge RC (2006) Novel high-affinity PPARgamma agonist alone and in combination with paclitaxel inhibits human anaplastic thyroid carcinoma tumor growth via p21WAF1/ CIP1. Oncogene 25:2304-2317

10. Shimazaki N, Togashi N, Hanai M, Isoyama T, Wada K, Fujita T, Fujiwara K, Kurakata S (2008) Anti-tumour activity of CS7017, a selective peroxisome proliferator-activated receptor gamma agonist of thiazolidinedione class, in human tumour xenografts and a syngeneic tumour implant model. Eur J Cancer 44:1734-1743

11. Nakajima TE, Yamada Y, Hamano T, Furuta K, Matsuda T, Fujita S (2010) Adipocytokines as new promising markers of colorectal tumors: adiponectin for colorectal adenoma, and resistin and visfatin for colorectal cancer. Cancer Sci 101:1286-1291

12. Pishvaian MJ, Marshall JL, Wagner AJ, Hwang JJ, Malik S, Cotarla I, Deeken JF, He AR, Daniel H, Halim AB, Zahir H, Copigneaux C, Liu K, Beckman RA, Demetri GD (2012) A phase 1 study of efatutazone, an oral peroxisome proliferator-activated receptor gamma agonist, administered to patients with advanced malignancies. Cancer 118:5403-5413

13. Tournigand C, André T, Achille E, Lledo G, Flesh M, Mery-Mignard D, Quinaux E, Couteau C, Buyse M, Ganem G, Landi B, Colin P, Louvet C, de Gramont A (2004) FOLFIRI followed by FOLFOX6 or the reverse sequence in advanced colorectal cancer: a randomized GERCOR study. J Clin Oncol 22:229-237

14. Muro K, Boku N, Shimada Y, Tsuji A, Sameshima S, Baba $H$, Satoh $T$, Denda $T$, Ina $K$, Nishina $T$, Yamaguchi $K$, Takiuchi H, Esaki T, Tokunaga S, Kuwano H, Komatsu Y, Watanabe M, Hyodo I, Morita S, Sugihara K (2010) Irinotecan plus S-1 (IRIS) versus fluorouracil and folinic acid plus irinotecan (FOLFIRI) as second-line chemotherapy for metastatic colorectal cancer: a randomized phase $2 / 3$ noninferiority study (FIRIS study). Lancet Oncol 11:853-860

15. Maeda N, Takahashi M, Funahashi T, Kihara S, Nishizawa H, Kishida K, Nagaretani H, Matsuda M, Komuro R, Ouchi N, Kuriyama H, Hotta K, Nakamura T, Shimomura I, Matsuzawa Y (2001) PPARgamma ligands increase expression and plasma concentrations of adiponectin, an adipose-derived protein. Diabetes 50: 2094-2099 